\title{
Sistemas de cultivo de arroz irrigado e a compactação de um Planossolo(1)
}

\author{
Alceu Pedrotti( ${ }^{(2)}$, Eloy Antonio Pauletto ${ }^{(3)}$, Algenor da Silva Gomes ${ }^{(4)}$, Ariovaldo Luis Turatti( ${ }^{(3)}$ e Silvio Crestana ${ }^{(5)}$
}

Resumo - Em 1993 foi avaliada a compactação de um Planossolo cultivado sob diferentes sistemas de manejo, medindo-se a densidade do solo, por meio do método do anel volumétrico, em três camadas de solo. Os tratamentos consistiram de seis sistemas de manejo, selecionados de um experimento conduzido na Embrapa-Centro de Pesquisa Agropecuária de Clima Temperado, desde 1985. Observaram-se, de maneira geral, valores altos de densidade do solo; na primeira camada de solo $(0-10 \mathrm{~cm})$ o sistema que apresentou o menor valor foi o cultivo de arroz em plantio direto sob a resteva do azevém e nos sistemas que envolvem maior mobilização do solo (arroz contínuo e rotação arroz-soja-milho) os valores de densidade do solo foram maiores em relação aos sistemas de preparo reduzido do solo (azevém x arroz plantio direto e soja $\mathrm{x}$ arroz plantio direto). Ocorreu maior compactação, em relação à primeira camada, na camada intermediária $(10-20 \mathrm{~cm})$, em todos os sistemas de cultivo, exceto no sistema de rotação arroz-soja-milho, no qual não houve diferença de densidade entre as camadas de solo.

Termos para indexação: várzeas, densidade do solo, manejo do solo, fatores edáficos.

Tillage systems of irrigated rice and compaction of a Planosol

\begin{abstract}
In 1993, it was evaluated the compaction of a Planosol (Albaqualf) submitted to different tillage systems, measuring soil density through the volumetric ring method, in three layers. Treatments consisted of six tillage systems selected from an experiment conducted at Embrapa-Centro de Pesquisa Agropecuária de Clima Temperado, Pelotas, RS, Brazil, since 1985. High soil density values were obtained; in the first layer $(0-10 \mathrm{~cm})$, the no-tillage rice cultivation on the rye-grass straw was the system with the lowest value. The systems that involved more soil mobilization (rice continuous and rotation rice-soybeans-maize) resulted in higher soil density values compared to the reduced preparing systems (rye-grass $\mathrm{x}$ rice in zero tillage and soybean $\mathrm{x}$ rice in zero tillage). Compacted structures were identified in the intermediate layer $(10-20 \mathrm{~cm})$ in all tillage systems, except for the rotation ricesoybean-maize, where soil density differences were not detected among soil layers.
\end{abstract}

Index terms: lowland, soil density, soil management, edaphic factors.

(1) Aceito para publicação em 31 de outubro de 2000. Extraído da dissertação de mestrado apresentada pelo primeiro autor à Faculdade de Agronomia Eliseu Maciel (FAEM), Universidade Federal de Pelotas (UFPel), RS.

(2) Universidade Federal de Sergipe, Dep. de Engenharia Agronômica, Av. Mal. Rondon, s/n, Campus Universitário, Jardim Rosa Elze, CEP 49100-000 São Cristovão, SE.

E-mail: apedroti@ufs.br

(3) UFPel, FAEM, Dep. de Solos, Caixa Postal 354, CEP 96010-900 Pelotas, RS.

E-mail: pauletto@uppeltchê.br

(4) Embrapa-Centro de Pesquisa Agropecuária de Clima Temperado, Caixa Postal 403, CEP 96001-970 Pelotas, RS.

E-mail: algenor@cpact.embrapa.br

(5) Embrapa-Centro Nacional de Pesquisa e Desenvolvimento de Instrumentação Agropecuária (CNPDIA), Caixa Postal 741, CEP 14000-000 São Carlos, SP.

\section{Introdução}

Os solos de várzea do sul do Brasil, apesar das grandes variações, apresentam uma característica comum, que é a sua formação sob condições de hidromorfismo (Gomes et al., 1992). Esses solos são encontrados nas planícies dos rios e lagos, onde se desenvolvem sobre sedimentos. Como estes sedimentos apresentam grande variabilidade na composição granulométrica e mineralógica, os solos desenvolvidos deles apresentam grande variação de características de um local para outro, que se refletem 
na aptidão de seu uso (Klamt et al., 1985). No Rio Grande do Sul, estes solos abrangem cerca de sete milhões de hectares, correspondendo a $25 \%$ da área total do Estado, com relevo plano a suavemente ondulado (Gomes et al., 1992).

O cultivo continuado do arroz em uma mesma área leva à autolimitação da cultura, isto é, acaba impedindo a continuidade do cultivo, devido ao aumento da infestação por plantas daninhas, em especial o arroz vermelho. A intensificação do uso de veículos e implementos agrícolas pesados, utilizados para o preparo convencional dos solos de várzea, agrava ainda mais os problemas de estrutura já existentes nesses tipos de solos. Associados a ações sucessivas de preparo ao longo dos anos, podem trazer sérios problemas de drenagem, assim como promover a compactação subsuperficial, dificultando a movimentação da água e a aeração nesses solos (Pauletto et al., 1993).

Tem-se buscado alternativas de manejo dos solos cultivados com o arroz irrigado que sejam, ao mesmo tempo, mais eficientes no controle do arroz vermelho, economicamente mais viáveis e capazes de propiciarem o uso mais intensivo do solo, sem contudo comprometer o seu já precário estado (Gomes et al., 1992).

Conforme Centurion \& Demattê (1992a, 1992b), a intensidade da compactação em diferentes sistemas de preparo e cultivo do solo varia com o tipo e a profundidade de atuação dos implementos agrícolas utilizados. Vários parâmetros podem ser utilizados para caracterizar o grau de compactação como a densidade e a porosidade do solo, que são comumente empregadas em análises de laboratório (Forsythe, 1975; Beltrame \& Taylor, 1980; Grant \& Lafond, 1993).

Para avaliar o comportamento de solos de várzea em relação aos diferentes sistemas de cultivo, a densidade do solo é um dos mais importantes parâmetros físicos para caracterizar e avaliar os efeitos de diferentes sistemas de manejo na compactação do solo (Pedrotti \& Dias Júnior, 1996). A densidade do solo reflete o arranjamento das partículas do solo, que, por sua vez, define as características do sistema poroso. Assim, todas as manifestações que influenciam a disposição das partículas do solo afetarão diretamente os valores da densidade, sendo esta expressa normalmente em $\mathrm{Mg} / \mathrm{m}^{3}$. Em solo não-expansivo, este atributo físico varia em razão da textura, conteúdo de matéria orgânica e do grau de compactação do solo (Baver et al., 1972; Kiehl, 1979; Reichardt, 1990). De acordo com Kiehl (1979), o manejo inadequado do solo e o uso indiscriminado de máquinas agrícolas pesadas aumentam a densidade pela compactação dos horizontes superficiais do solo.

Com base nos dados fornecidos pela literatura (Abrão et al., 1979; Fernandes et al., 1983; Sidiras et al., 1984; Eltz et al., 1989; Raper et al., 1994; Albuquerque et al., 1995), pode-se observar que os diferentes sistemas de manejo de solo afetam diferentemente tanto a densidade do solo como a resistência mecânica à penetração. Para cada caso há a necessidade de um estudo pormenorizado, principalmente em solos de várzea e na cultura do arroz irrigado, mais especialmente nas condições edafoclimáticas do Rio Grande do Sul, onde as informações ainda são escassas.

Este trabalho teve o objetivo de avaliar o estado de compactação de um Planossolo submetido a diferentes sistemas de cultivo por meio da densidade do solo obtida pelo método do anel volumétrico.

\section{Material e Métodos}

O presente trabalho foi realizado em um experimento que vem sendo conduzido desde 1985, na Estação Experimental de Terras Baixas, da Embrapa-Centro de Pesquisa Agropecuária de Clima Temperado, localizado no Município de Capão do Leão, na planície costeira do Rio Grande do Sul, cujas coordenadas geográficas são: $31^{\circ} 52^{\prime} 0^{\prime \prime}$ de latitude Sul e 52 21 '24" de longitude Oeste, com altitude média aproximada de 13,2 m. O solo da área do experimento, segundo Fundação IBGE (1986), foi classificado como Planossolo Solódico, com argila de atividade alta, textura média argilosa com relevo plano, pertencente à Unidade de Mapeamento Pelotas, e classificado segundo Estados Unidos (1990) como um Albaqualf. Os valores de algumas características físicas do perfil modal são mostrados na Tabela 1. Os tratamentos utilizados neste trabalho foram os seguintes: $T_{1}$ ) sistema tradicional de cultivo de arroz irrigado: um ano arroz com preparo convencional, seguido de dois anos com pousio da área; $\mathrm{T}_{2}$ ) sistema de cultivo contínuo de arroz, com preparo convencional; $\mathrm{T}_{3}$ ) sistema de cultivo de arroz com rotação de culturas, com preparo convencional: arroz x soja x milho; $\mathrm{T}_{4}$ ) sucessão de culturas: azevém no inverno $\mathrm{x}$ arroz no verão em sistema de semeadura direta; $T_{5}$ ) sucessão de culturas: um ano soja no 
sistema convencional $\mathrm{x}$ arroz no sistema de semeadura direta e $\mathrm{T}_{6}$ ) testemunha: solo mantido nas condições naturais.

O cronograma de execução dos tratamentos que compõem o experimento nas diferentes safras agrícolas é mostrado na Tabela 2. As parcelas, em três repetições para cada tratamento, possuem uma área individual de $600 \mathrm{~m}^{2}$ (20 x $30 \mathrm{~m})$, com faixa de bordadura de $5 \mathrm{~m}$.

A condução do experimento foi feita de acordo com os diferentes sistemas de cultivo e culturas. Para o preparo convencional, realizou-se uma aração, normalmente no mês de outubro, seguida de uma ou duas gradagens, sendo a semeadura feita com plantadora convencional. A semeadura direta foi realizada utilizando-se máquina apropriada para este fim, após a dessecação do azevém no $\mathrm{T}_{4} \mathrm{e}$ da flora de sucessão no $T_{5}$ com herbicida de ação total. A adubação do experimento foi feita com base nos resultados obtidos na análise de solo, coletado nas parcelas correspondentes a cada tratamento e das exigências nutricionais das diferentes culturas instaladas. O controle das plantas daninhas, das pragas e de doenças, foi realizado conforme a necessidade e a colheita das culturas conforme as técnicas convencionais.
A compactação do solo nos diferentes sistemas de cultivo foi avaliada mediante a determinação da densidade do solo pelo método do anel volumétrico, segundo Kiehl (1979). No final da safra agrícola 92/93 (maio a setembro de 1993), coletaram-se amostras de acordo com método denominado de Método Hidráulico de Amostragem Indeformada do Solo (MHAIS), utilizando-se canos de PVC (Figura 1), com, aproximadamente, $90 \mathrm{~cm}^{3}$ $(\varnothing \cong 9,80 \mathrm{~cm}$ e $\mathrm{h} \cong 10 \mathrm{~cm})$, nas profundidades médias de 0-10, 10-20 e 20-30 cm, em três locais distintos de cada parcela, com três repetições e encaminhadas ao Laboratório de Física do Solo da Faculdade de Agronomia Eliseu Maciel, UFPel, para determinação da massa do solo seco em estufa a $105^{\circ} \mathrm{C}$ por 24 horas. O cálculo da densidade do solo foi feito dividindo-se a massa do solo seco pelo volume do anel.

Durante a coleta, para que houvesse mínima perturbação na amostra, os tubos foram introduzidos no solo com auxílio de um macaco hidráulico automotivo. Optou-se por este equipamento por ser leve, de fácil utilização, e com possibilidade de aplicação da carga de modo suave. Após a remoção da vegetação ou restos de cultura do local de coleta, foram introduzidos no solo dois trados amostradores do tipo helicoidal, e acoplada uma travessa

Tabela 1. Profundidade, densidade do solo, composição granulométrica e classe textural nos diferentes horizontes do perfil característico do Planossolo.

\begin{tabular}{lcccccl}
\hline Horizonte & $\begin{array}{c}\text { Profundidade } \\
(\mathrm{cm})\end{array}$ & $\begin{array}{c}\text { Densidade } \\
\left(\mathrm{Mg} / \mathrm{m}^{3}\right)\end{array}$ & \multicolumn{3}{c}{ Granulometria $(\mathrm{g} / \mathrm{kg})$} & Classe textural \\
\cline { 4 - 6 } & $0-20$ & 1,45 & 423 & 478 & 98 & Franco \\
do Planossolo
\end{tabular}

Fonte: Vasconcelos (1993).

Tabela 2. Cronograma do experimento para os diferentes sistemas de cultivo nas diferentes safras agrícolas.

\begin{tabular}{cllllllll}
\hline Tratamento $^{(1)}$ & \multicolumn{7}{c}{ Safra } \\
\cline { 2 - 9 } & $85 / 86$ & $86 / 87$ & $87 / 88$ & $88 / 89$ & $89 / 90$ & $90 / 91$ & $91 / 92$ & $92 / 93$ \\
\hline $\mathrm{T}_{1}$ & Arroz & Pousio & Pousio & Arroz & Pousio & Pousio & Arroz & Pousio \\
$\mathrm{T}_{2}$ & Arroz & Arroz & Arroz & Arroz & Arroz & Arroz & Arroz & Arroz \\
$\mathrm{T}_{3}$ & Arroz & Soja & Milho & Arroz & Soja & Milho & Arroz & Soja \\
$\mathrm{T}_{4}$ & Arroz & Azevém & Arroz & Azevém/ & Azevém/ & Azevém/ & Azevém/ & Azevém/ \\
& & & Arroz & Arroz & Arroz & Arroz & Arroz \\
$\mathrm{T}_{5}$ & Arroz & Soja & Arroz & Soja & Arroz & Soja & Arroz & Soja \\
$\mathrm{T}_{6}$ & \multicolumn{2}{l}{ Solo mantido sem cultivo } & & & & & \\
\hline
\end{tabular}

${ }^{(1)} \mathrm{T}_{1}$ : sistema tradicional; $\mathrm{T}_{2}$ : arroz contínuo; $\mathrm{T}_{3}$ : rotação arroz $\mathrm{x}$ soja $\mathrm{x}$ milho; $\mathrm{T}_{4}$ : azevém $\mathrm{x}$ arroz em plantio direto; $\mathrm{T}_{5}$ : soja $\mathrm{x}$ arroz em plantio direto; $\mathrm{T}_{6}$ : testemunha. 


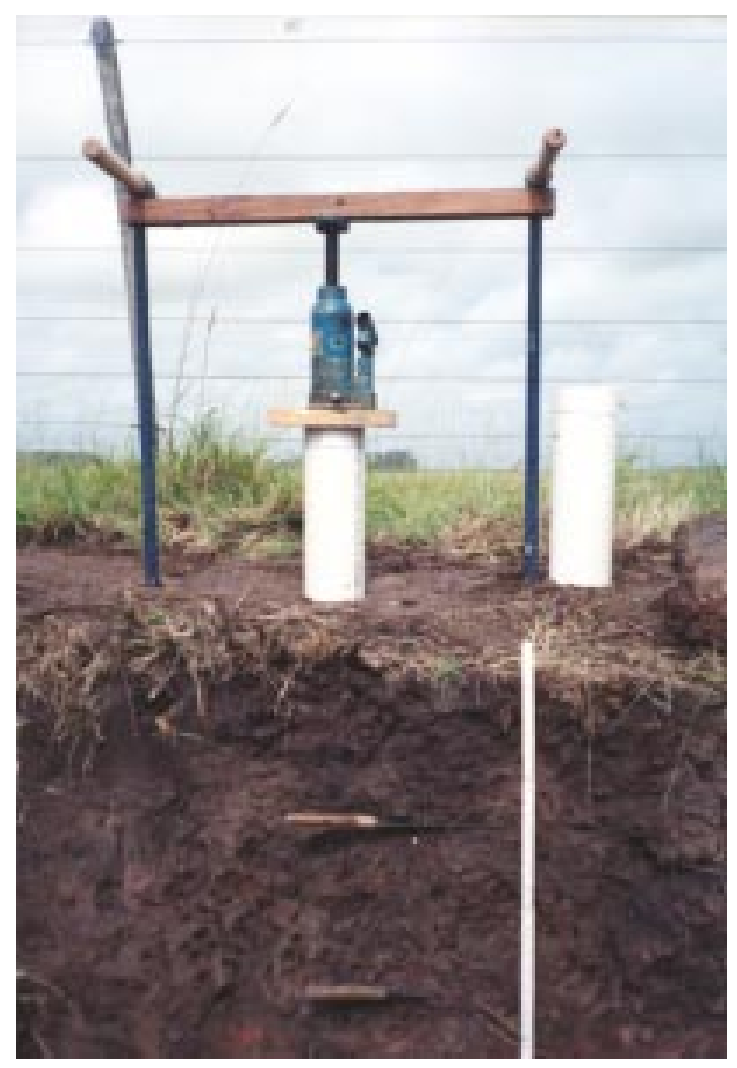

Figura 1. Vista do conjunto amostrador hidráulico do método hidráulico de amostragem indeformada do solo (MHAIS), utilizado no presente estudo.

chanfrada de madeira na parte superior dos trados. Este sistema serviu como sustentação para o acionamento do macaco hidráulico quando da introdução do tubo de PVC no solo. Através do acionamento do macaco hidráulico possibilitou-se a introdução lenta e gradual do tubo evitando qualquer possível perturbação da amostra. A Figura 1 mostra o conjunto do MHAIS, para a coleta das amostras indeformadas de solo.

Para a retirada do tubo com a amostra, utilizou-se uma cordoalha fina de aço que foi amarrada (laçada) ao tubo e tracionada manualmente. Em seguida, fez-se a limpeza do tubo e impermeabilizaram-se as duas extremidades com parafina líquida a $60^{\circ} \mathrm{C}$. Colocou-se, ainda, um tampão nas extremidades, para a manutenção da integridade física das amostras. No Laboratório de Física do Solo do Departamento de Solos da UFPel, marcaram-se, a cada $10 \mathrm{~cm} \mathrm{de}$ comprimento, e seccionaram-se, com serra manual (destinada para materiais metálicos) as amostras em três partes, e avaliou-se, em cada uma das profundidades médias, a massa de solo seco em estufa a $105^{\circ} \mathrm{C}$ por, no mínimo, 48 horas.

Os resultados de densidade do solo, obtidos através do Método do Anel Volumétrico, foram submetidos à análise de variância, e as médias, comparadas entre si pelo teste de Duncan a $5 \%$ de probabilidade.

\section{Resultados e Discussão}

A análise de variância aplicada aos referidos valores mostra que houve diferença significativa em tratamentos, profundidades e na interação destes dois fatores, mostrando que os tratamentos afetam a densidade ao longo do perfil do solo (Tabela 3).

Analisando-se o efeito dos diferentes tratamentos na densidade do solo, na profundidade de 0-10 cm, observa-se que os tratamentos $T_{2}$ e $T_{3}$ e a testemunha foram os que apresentaram os maiores valores, não diferindo estatisticamente entre si (Tabela 4). Na profundidade de 10-20 cm, $\mathrm{T}_{2}$ foi superior $\mathrm{a} \mathrm{T}_{1} \mathrm{e}$ à testemunha. A presença desta camada compactada na profundidade de $10-20 \mathrm{~cm}$, denominada de pé-de-grade, principalmente nos sistemas que empregam o preparo convencional do solo em virtude da ação contínua dos implementos e ao trânsito de máquinas agrícolas, também foi relatada por Centurion \& Demattê (1992a, 1992b), Hammel (1989) citado por Grant \& Lafond (1993) e Raper et al. (1994). Na profundidade de $20-30 \mathrm{~cm}, \mathrm{~T}_{2}$ foi maior do que $\mathrm{T}_{5}$ mas não diferiu dos demais (Tabela 4). Estes resultados evidenciam que os sistemas que envolvem maior mobilização do solo $\left(\mathrm{T}_{2}\right.$ e $\left.\mathrm{T}_{3}\right)$ levam à obtenção de valores mais elevados de densidade, quando comparados com os sistemas que utilizam o preparo reduzido do solo $\left(\mathrm{T}_{4}\right.$ e $\left.\mathrm{T}_{5}\right)$.

Observa-se, também, que a variação da densidade do solo nos diferentes sistemas de manejo foi mais pronunciada na camada superficial (Tabela 4). Resultados semelhantes foram encontrados por Sidiras et al. (1984) e Centurion \& Demattê (1992a, $1992 b)$. O sistema de rotação de culturas $\left(T_{3}\right)$ foi o único em que não ocorreu variação entre os valores de densidade do solo nas três camadas estudadas, apresentando, portanto, o perfil mais homogêneo.

O menor valor de densidade do solo na camada superficial foi observado no sistema de plantio direto de arroz na resteva do azevém. Estes resultados provavelmente estão associados ao uso do próprio 
sistema de cultivo (azevém no inverno seguido de arroz no verão - $\mathrm{T}_{4}$ ), o que proporciona menor revolvimento e maior cobertura do solo o ano todo, e, conseqüentemente, menor compactação, além do denso sistema radicular característico da cultura do azevém. Estas constatações vão ao encontro dos dados obtidos por Hammel (1989) citado por Albuquerque et al. (1995). Este comportamento pode ser atribuído também à possível consolidação natural do solo, causada pelo rearranjamento natural que o solo tende a apresentar quando deixa de sofrer manipulação mecânica (mobilização). Entretanto, com o passar dos anos, é de se esperar que os valores de densidade do solo decresçam, devido, em parte, ao aumento do teor de matéria orgânica incorporada na camada superficial que favorece um maior desenvolvimento da estrutura do solo (Fernandes et al., 1983;

Tabela 3. Análise de variância dos valores de densidade do solo, obtidos pelo método do anel volumétrico.

\begin{tabular}{lrccrl}
\hline $\begin{array}{l}\text { Causa da } \\
\text { variação }\end{array}$ & GL & SQ & QM & F & P>F \\
\hline Bloco & 8 & 0,036 & 0,004 & 1,13 & 0,350 \\
Tratamento & 5 & 0,194 & 0,039 & 9,73 & $0,000^{* *}$ \\
$\begin{array}{l}\text { Profundidade } \\
\text { Tratamentos x }\end{array}$ & 2 & 0,463 & 0,232 & 58,14 & $0,000^{* *}$ \\
$\quad$ profundidade & 10 & 0,154 & 0,015 & 3,87 & $0,000^{* *}$ \\
Resíduo & 136 & 0,542 & 0,004 & - & \\
\hline Total & 161 & 1,390 & - & - & \\
\hline
\end{tabular}

**Significativo a $1 \%$ de probabilidade
Albuquerque et al., 1995). Portanto, a grande quantidade de matéria seca do azevém deixada na superfície ou parcialmente incorporada, e o denso sistema radicular desta cultura podem ter contribuído para uma menor densidade do solo sob o sistema de plantio direto de arroz. Todos estes fatos podem ter colaborado para a obtenção da interação altamente significativa entre tratamentos (sistemas de preparo de solo e cultivo de arroz irrigado) e profundidade do solo na análise de variância da densidade do solo (Tabela 3).

$\mathrm{Na}$ literatura encontram-se controvérsias sobre o efeito do sistema de plantio direto na densidade do solo; a maioria dos registros refere-se a solos de terras altas. Vieira et al. (1978), Abrão et al. (1979) e Fernandes et al. (1983) encontraram valores de densidade da camada superficial de um solo no sistema de plantio direto, superiores à do sistema de manejo convencional. Por outro lado, os trabalhos de Machado \& Brum (1978), Sidiras et al. (1984), Vieira \& Muzilli (1984), Eltz et al. (1989), Chang \& Lindwall (1990), Hammel (1989) citado por Grant \& Lafond (1993) e Gantzer \& Blake (1978) citados por Lal et al. (1994) encontraram valores de densidade do solo maiores no sistema convencional do que no sistema de plantio direto.

O solo, neste sistema, sofreu maior compactação nesta camada, provavelmente, em virtude das operações de preparo do solo (Oliveira, 1990), realizadas na cultura da soja no sistema convencional, e do tráfego de máquinas por ocasião do cultivo do arroz,

Tabela 4. Valores médios de densidade do solo $\left(\mathrm{Mg} / \mathrm{m}^{3}\right)$, obtidos pelo método do anel volumétrico nas profundidades e nos sistemas de cultivo estudados ${ }^{(1)}$.

\begin{tabular}{ccccccc}
\hline \multirow{2}{*}{$\begin{array}{c}\text { Profundidade } \\
(\mathrm{cm})\end{array}$} & \multicolumn{7}{c}{ Tratamento $^{(2)}$} \\
\cline { 2 - 6 } & $\mathrm{T}_{1}$ & $\mathrm{~T}_{2}$ & $\mathrm{~T}_{3}$ & $\mathrm{~T}_{4}$ & $\mathrm{~T}_{5}$ & $\mathrm{~T}_{6}$ \\
\hline $0-10$ & $1,58 \mathrm{bcB}$ & $1,68 \mathrm{aB}$ & $1,67 \mathrm{aA}$ & $1,47 \mathrm{~dB}$ & $1,56 \mathrm{cB}$ & $1,63 \mathrm{abB}$ \\
& $( \pm 0,056)$ & $( \pm 0,072)$ & $( \pm 0,063)$ & $( \pm 0,106)$ & $( \pm 0,040)$ & $( \pm 0,044)$ \\
$10-20$ & $1,67 \mathrm{bA}$ & $1,75 \mathrm{aA}$ & $1,71 \mathrm{abA}$ & $1,68 \mathrm{abA}$ & $1,69 \mathrm{abA}$ & $1,66 \mathrm{bB}$ \\
& $( \pm 0,075)$ & $( \pm 0,057)$ & $( \pm 0,067)$ & $( \pm 0,096)$ & $( \pm 0,051)$ & $( \pm 0,039)$ \\
$20-30$ & $1,72 \mathrm{abA}$ & $1,76 \mathrm{aA}$ & $1,73 \mathrm{abA}$ & $1,72 \mathrm{abA}$ & $1,68 \mathrm{bA}$ & $1,73 \mathrm{abA}$ \\
& $( \pm 0,028)$ & $( \pm 0,057)$ & $( \pm 0,063)$ & $( \pm 0,045)$ & $( \pm 0,083)$ & $( \pm 0,053)$ \\
& & & & & & \\
Média & 1,65 & 1,73 & 1,70 & 1,63 & 1,65 & 1,67 \\
\hline
\end{tabular}

(1)Médias seguidas de mesma letra, minúscula na linha e maiúscula na coluna, não diferem pelo teste de Duncan a 5\% de probabilidade; os dados representam médias de 27 repetições e os números entre parêntesis representam os desvios padrões. ${ }^{(2)} \mathrm{T}_{1}$ : sistema tradicional; $\mathrm{T}_{2}$ : arroz contínuo; $\mathrm{T}_{3}$ : rotação arroz $\mathrm{x}$ soja $\mathrm{x}$ milho; $\mathrm{T}_{4}$ : azevém $\mathrm{x}$ arroz em plantio direto; $\mathrm{T}_{5}$ : soja $\mathrm{x}$ arroz em plantio direto; $\mathrm{T}_{6}$ : testemunha. 
no sistema de plantio direto (Machado et al., 1981; Eltz et al., 1989; Gomes et al., 1992).

Os valores obtidos de densidade do solo nos diferentes tratamentos e profundidades estudadas (Tabela 4) estão relativamente altos, em comparação com os apresentados por Reichardt (1990), e acima da faixa encontrada em solos minerais (Hillel, 1970, citado por Anjos et al., 1994), o que é uma característica do Planossolo como ressaltam os trabalhos de Gomes et al. (1992), Peña (1993) e Pauletto et al. (1993). Entretanto, estes valores se aproximam dos valores encontrados em levantamentos e descrições de perfis característicos do solo do experimento (Tabela 1), conforme Gomes et al. (1992) e Vasconcelos (1993).

A maioria dos solos de várzea possuem naturalmente valores de densidade mais elevados, e esta condição adversa pode ser agravada quando o solo é submetido ao sistema de preparo convencional, comprometendo o rendimento da cultura, principalmente quando se quer introduzir culturas de sequeiro (Gomes et al., 1992).

O solo do presente experimento apresenta, na sua camada intermediária $(10-20 \mathrm{~cm})$, condições restritivas ao crescimento do sistema radicular de culturas de sequeiro, uma vez que, em todos os sistemas de cultivo, com exceção do $T_{3}$ (rotação arroz-soja-milho), ocorreu um crescimento significativo dos valores de densidade do solo (Tabela 4).

\section{Conclusões}

1. Os sistemas que envolvem maior mobilização do solo promovem maiores valores de densidade no perfil do solo.

2. O sistema de rotação de culturas e o sistema de plantio direto de arroz na resteva do azevém provocam as mais baixas e as mais altas possibilidades de ocorrência de pé-de-grade, respectivamente.

3. Os sistemas de cultivo que envolvem ação antrópica, de maneira geral, contribuem para o aumento dos valores de densidade do solo.

4. Os sistemas de preparo reduzido do solo favorecem as melhores condições físicas do solo em termos de densidade.

5. Os sistemas de preparo convencional de solo contribuem para a degradação física do solo, propiciando o surgimento de camadas compactadas.

\section{Agradecimentos}

Aos funcionários do Laboratório de Física do Solo da Universidade Federal de Pelotas e da EmbrapaCentro de Pesquisa Agropecuária de Clima Temperado, pela colaboração na condução do experimento; à Universidade Federal de Pelotas e à Embrapa-Centro de Pesquisa Agropecuária de Clima Temperado, pelo apoio financeiro.

\section{Referências}

ABRÃO, P. R.; GOEFERT, C. F.; GUERRA, M.; ELTZ, F. L. F.; CASSOL, E. A. Efeito de sistemas de preparo do solo sobre características de um Latossolo Roxo distrófico. Revista Brasileira de Ciência do Solo, Campinas, v. 3, n. 3, p. 169-172, 1979.

ALBUQUERQUE, J. A.; REINERT, D. J.; FIORIN, J. E.; RUEDELL, J.; PETRERE, C.; FONTINELLI, F. Rotação de culturas e sistemas de manejo de solo: efeito sobre a forma de estrutura do solo ao final de sete anos. Revista Brasileira de Ciência do Solo, Campinas, v. 19, p. 115119, 1995.

ANJOS, J. T.; UBERTI, A. A. A.; VIZZOTTO, V. J.; LEITE, G. B.; KRIEGER, M. Propriedades físicas em solos sob diferentes sistemas de uso e manejo. Revista Brasileira de Ciência do Solo, Campinas, v. 18, p. 139145, 1994.

BAVER, L. D.; GARDNER, W. H.; GARDNER, W. H. Física del suelo. México : Hispano Americano, 1972. $529 \mathrm{p}$.

BELTRAME, L. F. C.; TAYLOR, F. C. Causas e efeitos da compactação do solo. Lavoura Arrozeira, Porto Alegre, v. 33, n. 318, p. 59-62, jan./fev. 1980.

CENTURION, J. F.; DEMATTÊ, J. L. I. Efeito de sistemas de preparo nas propriedades físicas de um solo sob cerrado cultivado com soja. Revista Brasileira de Ciência do Solo, Campinas, v. 9, n. 3, p. 263-266, 1992a.

CENTURION, J. F.; DEMATTÊ, J. L. I. Sistemas de preparo de solo de cerrado: efeito nas propriedades físicas e na cultura do milho. Pesquisa Agropecuária Brasileira, Brasília, v. 27, n. 2, p. 315-324, fev. 1992 b.

CHANG G.; LINDWALL, C. W. Comparison of the effect of long term tillage and crop rotation on physical properties of a soil. Canadian Journal of Soil Science, Ottawa, v. 32, p. 53-55, 1990. 
ELTZ, F. L. F.; PEIXOTO, R. T. G.; JASTER, F. Efeitos de sistemas de preparo do solo nas propriedades físicas e químicas de um Latossolo Bruno Álico. Revista Brasileira de Ciência do Solo, Campinas, v. 13, p. 259-267, 1989.

ESTADOS UNIDOS. Department of Agriculture. Soil Survey Staff. Keys to soil taxonomy. 4. ed. Blacksburg : Pocahontas, 1990. 422 p. (Soil Management Support Services Technical Monograph, 6)

FERNANDES, B.; GALLOWAY, H. M.; BRONSON, R. D.; MANNERING, J. V. Efeito de três sistemas de preparo do solo na densidade aparente, na porosidade total $\mathrm{e}$ na distribuição dos poros, em dois solos (Typic Argiaquoll e Typic Hapludalf). Revista Brasileira de Ciência do Solo, Campinas, v. 7, n. 3, p. 329-333, 1983.

FORSYTHE, W. Física de suelos: manual de laboratorio. San José : IICA, 1975. 212 p.

FUNDAÇÃO IBGE (Rio de Janeiro, RJ). Folha SH.22 Porto Alegre e parte das folhas SH.21 Uruguaiana e SI.22 Lagoa Mirim. Rio de Janeiro, 1986. 796 p. (Levantamento de Recursos Naturais, 33).

GOMES, A. S.; CUNHA, N. G.; PAUlETtO, E. A.; SILVEIRA, R. J. C.; TURATTI, A. L. Solos de várzea: uso e manejo. In.: MARCÂNTONIO, G. (Coord.). Solos e irrigação. Porto Alegre : Ed. da UFRGS/FEDERACITE, 1992. p. 64-79.

GRANT, C. A.; LAFOND, G. P. The effects of tillage systems and crop sequences on soil bulk density and penetration resistance on a clay soil in southern Saskatchewan. Canadian Journal of Soil Science, Ottawa, v. 73, p. 223-232, May 1993.

KIEHL, E. J. Manual de edafologia: relação solo-planta. São Paulo : Agronômica Ceres, 1979. 273 p.

KLAMT, E.; KAMPF, N.; SCHENEIDER, P. Solos de várzea no Estado do Rio Grande do Sul. Porto Alegre : UFRGS, 1985. (Boletim Técnico de Solos, 4).

LAL, R.; MAHBOUBI, A. A.; FAUSEY, N. R. Long-term tillage and rotation effects on properties of a central Ohio soil. Soil Science Society of America Journal, Madison, v. 58, p. 517-522, Mar./Apr. 1994

MACHADO, J. A.; BRUM, A. C. R. Efeito de sistemas de cultivo em algumas propriedades físicas do solo. Revista Brasileira de Ciência do Solo, Campinas, v. 2, n. 2 , p. $81-84,1978$.
MACHADO, J. A.; SOUZA, D. M. P.; BRUM, A. C. R. Efeito de anos de cultivo convencional em propriedades físicas do solo. Revista Brasileira de Ciência do Solo, Campinas, v. 5, n. 3, p. 187-189, 1981.

OLIVEIRA, E. F. Efeito do sistema de preparo do solo nas suas características físicas e químicas e no rendimento de trigo e soja em Latossolo Roxo: I. Resultados de pesquisa obtidos de 1982 a 1988. Cascavel : Organização das Cooperativas do Estado do Paraná, 1990. 54 p. (Resultados de Pesquisa, 4).

PAUlETTO, E. A.; GOMES, A. S.; FRANZ, A. F. H.; SOUZA, R. O. Manejo de solo e água em arroz irrigado. In: PESKE, S.; NEDEL, J.; BARROS, A. (Ed.). Produção de sementes de arroz. Pelotas : Ed. da UFPel, 1993. v. 1 , p. $64-144$.

PEDROTTI, A.; DIAS JÚNIOR, M. S. Compactação do solo: como evitá-la. Agropecuária Catarinense, Florianópolis, v. 9, n. 4, p. 50-52, dez. 1996.

PEÑA, Y. A. Efeito de diferentes sistemas de cultivo sobre atributos físicos de um solo de várzea, cultivado com arroz irrigado. Pelotas : UFPel-FAEM, 1993. 97 p. Dissertação de Mestrado.

RAPER, R. L.; REEVES, D. W.; BURTH, E. C.; TORBERT, H. A. Conservation tillage and traffic effects on soil condition. Transactions of the ASAE, St. Joseph, v. 37, n. 3, p. 763-768, 1994.

REICHARDT, K. A água em sistemas agrícolas. São Paulo : Manole, 1990. $171 \mathrm{p}$.

SIDIRAS, N.; VIEIRA, S. R.; ROTH, C. H. Determinação de algumas características físicas de um Latossolo Roxo distrófico sob plantio direto e preparo convencional. Revista Brasileira de Ciência do Solo, Campinas, v. 8, p. 265-268, 1984.

VASCONCELOS, E. B. de. Levantamento dos atributos físicos e hídricos de três solos de várzea do Rio Grande do Sul. Pelotas : UFPel-FAEM, 1993. 79 p. Dissertação de Mestrado.

VIEIRA, M. J.; COGO, N. P.; CASSOL, E. A. Perdas por erosão em diferentes sistemas de preparo do solo para a cultura da soja (Glycine max. (L.) Merril) em condições de chuva simulada. Revista Brasileira de Ciência do Solo, Campinas, v. 2, n. 3, p. 209-214, 1978.

VIEIRA, M. J.; MUZILLI, O. Características físicas de um Latossolo Vermelho Escuro sob diferentes sistemas de manejo. Pesquisa Agropecuária Brasileira, Brasília, v. 19, n. 7 , p. $873-882$, jul. 1984. 